\title{
Concordancia de tres definiciones de síndrome metabólico en pacientes hipertensos
}

\section{Agreement between three definitions of metabolic syndrome in hypertensive patients}

Ángel J. Paternina-Caicedo' ${ }^{1}$, Gabriel Alcalá-Cerra², Jaime Paillier-Gonzales ${ }^{3}$, Ángela M. Romero-Zarante ${ }^{4}$ y Nelson Alvis-Guzmán ${ }^{5}$

1 Facultad de Medicina. Universidad de Cartagena. Colombia. angel.paternina@gmail.com

2 Clínica de la Costa, Barranquilla, Colombia. alcalagabriel@gmail.com

3 Hospital general de Medellín, IPS universitaria Clínica León XIII. Medellín, Colombia. paillier999@hotmail.com

4 Hospital San Francisco de Asís. Sincelejo, Colombia. anmaroza@hotmail.com

5 Facultad de Ciencias Económicas. Universidad de Cartagena. Colombia. nalvis@yahoo.com

Recibido 17 Abril 2009/Enviado para Modificación 31 Octubre 2009/Aceptado 15 Noviembre 2009

\section{RESUMEN}

Objetivo Determinar la concordancia entre tres definiciones de síndrome metabólico (SMet) en pacientes hipertensos colombianos.

Materiales y Métodos Se diseñó un estudio descriptivo en 323 pacientes hipertensos de Cartagena de Indias. Se realizó historia clínica, medición de tensión arterial, perímetro abdominal, lípidos séricos y glucosa sérica en ayunas. Se comparó la prevalencia de SMet según la definición del Adult Treatment Panel III (ATP III), la American Heart Association (AHA), la International Diabetes Federation (IDF), y la IDF modificada (perímetro abdominal $\geq 88 \mathrm{~cm}$ en varones). Se evaluó la concordancia mediante el test de Kappa media de Cohen.

Resultados El 77,5\% de los pacientes fueron mujeres. La prevalencia global de SMet fue de $59,9 \%$ (ATP III), 83,6\% (AHA), 81,2\% (IDF) y 92.9\% (IDFm). La concordancia fue al menos clara en las comparaciones de definiciones, excepto la comparación de IDFm con ATP III y AHA. La mayor concordancia se obtuvo en la comparación IDF-AHA ( $\kappa$ $=0,638$ ).

Conclusiones La prevalencia de SMet fue más alta en pacientes hipertensos colombianos que en otros países. El análisis de concordancia demostró al menos, una clara concordancia la mayoría de las definiciones analizadas. Con una correlación pobre entre los criterios comparados con el IDFm (excepto con el IDF no modificado).

Palabras Clave: Síndrome X Metabólico, Dislipidemias, Hipertensión, Circunferencia Abdominal, Obesidad (fuente: DeCS, BIREME)

\section{ABSTRACT}

Objective Determining agreement between three definitions of metabolic syndrome (MS) in hypertensive Colombian patients. 
Materials and Methods A descriptive study of 323 hypertensive patients from Cartagena, Colombia, was designed. A medical history was compiled; waist circumference, serum lipids and fasting serum glucose were recorded. MS prevalence was compared by using by the definitions provided by the Adult Treatment Panel III (APT III), the American Heart Association (AHA), the International Diabetes Federation (IDF) and a modified IDF ( $\geq 88 \mathrm{~cm}$ waist circumference in males). Cohen's kappa test was used for evaluating agreement between means.

Results $77.5 \%$ of the patients were female. MS overall prevalence was $59.9 \%$ according to ATP III, $83.6 \%$ by AHA, $81.2 \%$ by IDF and $92.9 \%$ by IDFm. Agreement was fairly clear when comparing the definitions, except when comparing the IDFm one with ATP III and AHA. The greatest agreement was provided when comparing IDF to AHA ( $\kappa=0.638)$. Conclusions MS prevalence was higher in hypertensive Colombian patients than in other countries, probably because the greater prevalence of its components. Clear agreement was shown when comparing most definitions, apart from poor correlation between criteria compared using IDFm (except with non-modified IDF).

Key Words: Metabolic syndrome X, dyslipidaemia, hypertension, waist circumference, obesity (source: MeSH, NLM).

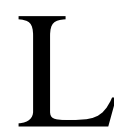

os criterios diagnósticos para el síndrome metabólico (SMet) han sido modificados reiteradamente tras la publicación de algunos estudios que demuestran diferencias importantes en el rendimiento de cada definición para detectar los individuos con alto riesgo cardiovascular. En 2001, el panel de adultos III del National Cholesterol Education Program (ATP-III) definió el SMet como se describe en la Tabla 1 (1). Posteriormente, esta fue modificada por un estamento conjunto entre el National Heart, Lung, and Blood Institute y la American Heart Assosiation (AHA), en la que fueron incluidos los pacientes con alteración en la glucosa en ayunas (glucemia $\geq 100 \mathrm{mg} / \mathrm{dL}$ ) o aquellos que no cumplieran los criterios metabólicos (hipertrigliceridemia, hiperglucemia o HDLc bajo) y/o que estuvieran recibiendo medicación para estas anormalidades (2). Según la más reciente definición de la International Diabetes Federation (IDF), además de la presencia de al menos dos de los siguientes criterios: glucemia en ayunas elevada o diagnóstico previo de diabetes mellitus, hipertrigliceridemia, colesterol HDL bajo, tensión arterial elevada, o tratamiento específico para alguna de estas anormalidades (Tabla 1); la presencia de obesidad central es un requisito indefectible para el diagnóstico del síndrome (3).

Los límites de perímetro abdominal (PA) que mejor se relacionan con un perfil aterogénico varían entre diferentes grupos étnicos; incluso dentro de Latinoamérica se han descrito diferencias importantes entre Méjico (4) y países de Suramérica (5-8), por lo que el PA a utilizarse en una determinada región debería ser derivado de datos locales. En Colombia, Pérez y colaboradores identificaron que en varones, un PAde 88 centímetros es el que mejor se relaciona 
con un perfil alto de riesgo cardiovascular (8); lo cual es similar a lo descrito en varones de Ecuador $(90 \mathrm{~cm})(6)$. Con base en estos estudios, el Consenso de Síndrome Metabólico de la Asociación Colombiana de Endocrinología ha aceptado la definición más reciente (9), en la cual se recomienda para Suramérica la utilización del PA derivado de los estudios realizados en el sur de Asia $(90 \mathrm{~cm}$ en hombres y $80 \mathrm{~cm}$ en mujeres).

En la región andina suramericana se ha descrito que los criterios de la IDF utilizando el PA recomendado para el Sur de Asia- son más sensibles que los del ATP-III para detectar la presencia de SMet en individuos que no han padecido eventos cardiovasculares (infarto agudo del miocardio, angina inestable, cirugía de revascularización coronaria o infarto cerebral); sin embargo, en aquellos que ya han tenido al menos un episodio, tal diferencia no existe (7).

La hipertensión arterial es la enfermedad cardiovascular más frecuente y varios estudios realizados fuera del continente americano (10-12) así como uno realizado en Colombia (13) han descrito diferencias clínicamente importantes entre la prevalencia de SMet en pacientes hipertensos en función de la definición empleada; sin embargo, el comportamiento de la concordancia entre las diferentes definiciones en esta población específica no ha sido determinado. En tal sentido, se planteó la presente investigación que buscó establecer la concordancia entre de las diferentes definiciones de SMet en pacientes hipertensos colombianos.

\section{MATERIALES Y MÉTODOS}

Se diseñó un estudio descriptivo de corte transversal en un grupo de pacientes con hipertensión arterial atendidos en la consulta ambulatoria de alto riesgo cardiovascular, en quienes se buscó establecer la concordancia entre de las diferentes definiciones de SMet.

\section{Población}

Se tomaron todos los pacientes ( $n=324)$ mayores de 25 años de ambos géneros con diagnóstico de hipertensión arterial que, entre los meses de enero y marzo de 2008, asistieron a la consulta ambulatoria de alto riesgo cardiovascular de los centros de atención especializada de la aseguradora Subsidiada Mutual Ser y que manifestaron participar voluntariamente en el estudio. Se excluyeron los pacientes con enfermedad crónica que altera los niveles de HDLc y/o triglicéridos (enfermedades del colágeno, hipercolesterolemia familiar); pacientes con enfermedades mentales que impidireon el correcto diligenciamiento del formulario; 
mujeres embarazadas, pacientes infectados por el virus de inmunodeficiencia adquirida, pacientes con historias clínicas incompletas.

Se diseño un protocolo de captura de información mediante un formulario para registrar datos relacionados con la presencia de dislipidemias, alteraciones en el metabolismo de los carbohidratos o medicación para alguna de estas anormalidades. Adicionalmente se documentó la medición sistemática de tensión arterial sistólica y diastólica, perímetro abdominal, glucosa en ayunas, colesterol total, HDL colesterol y triglicéridos en ayunas. Se determinó la tensión arterial sistólica y diastólica dos veces. El PA se determinó en la medida de las puntas de las crestas ilíacas, mientras el paciente respira normalmente. Las concentraciones séricas de glucosa, triglicéridos, colesterol total y HDLc fueron medidas tras un ayuno de por lo menos ocho horas mediante métodos estandarizados, por personal técnico y profesional idóneo, en un periodo no mayor de 2 meses antes del registro de los datos. Las mediciones fueron realizadas por personal especializado que labora en el programa de prevención y atención de enfermedades cardiovasculares de la aseguradora.

Síndrome metabólico. Para efectos del presente estudio se utilizaron las definiciones de SMet descritas en el Cuadro 1. Adicionalmente, se utilizó la definición de la IDF, considerando los parámetros utilizados en el sur de Asia para establecer la presencia de obesidad central, los cuales se aceptan por el Consenso de Síndrome Metabólico de la Asociación Colombiana de Endocrinología (9).

Cuadro 1. Definiciones de Síndrome Metabólico según el ATP III, la AHA y la IDF

\begin{tabular}{|c|c|c|c|}
\hline Criterios & ATP-III & $\mathrm{AHA}$ & IDF \\
\hline Prerrequisito & Ninguno & Ninguno. & Obesidad central \\
\hline $\begin{array}{l}\text { Criterios } \\
\text { requeridos }\end{array}$ & Al menos tres & Al menos tres & $\begin{array}{l}\text { Obesidad central y al } \\
\text { menos dos }\end{array}$ \\
\hline $\begin{array}{l}\text { Obesidad central } \\
\text { (PA) }\end{array}$ & $\begin{array}{l}\text { Hombres } \geq 102 \\
\text { cms. Mujeres: } \geq 88 \\
\text { cms. }\end{array}$ & $\begin{array}{l}\text { Hombres } \geq 102 \mathrm{cms} \text {. } \\
\text { Mujeres } \geq 88 \mathrm{cms} \text {. }\end{array}$ & $\begin{array}{l}\text { Hombres } \geq 90 \mathrm{cms} \text {. } \\
\text { Mujeres } \geq 80 \mathrm{cms} \text {. }\end{array}$ \\
\hline $\begin{array}{l}\text { Alteración en } \\
\text { metabolismo de } \\
\text { carbohidratos }\end{array}$ & $\begin{array}{l}\text { Glucosa en ayunas } \\
\geq 110 \mathrm{mg} / \mathrm{dL}\end{array}$ & $\begin{array}{l}\text { Glucemia en ayunas } \\
\geq 100 \mathrm{mg} / \mathrm{dL} \cdot \dagger\end{array}$ & $\begin{array}{l}\text { Glucemia en ayunas } \\
\geq 100 \mathrm{mg} / \mathrm{dL}{ }^{\circ}\end{array}$ \\
\hline Triglicéridos & $\geq 150 \mathrm{mg} / \mathrm{dL}$ & $\geq 150 \mathrm{mg} / \mathrm{dL} \dagger$. & $\geq 150 \mathrm{mg} / \mathrm{dL} \dagger$. \\
\hline $\begin{array}{l}\text { Tensión arterial } \\
\text { alta }\end{array}$ & $\geq 135 / 85 \mathrm{mmHg}$ & $\geq 135 / 85 \mathrm{mmHg}^{*}$ & $\geq 135 / 85 \mathrm{mmHg}^{*}$ \\
\hline $\begin{array}{l}\text { HDL colesterol } \\
\text { bajo }\end{array}$ & $\begin{array}{l}\text { Hombres: } \leq 40 \\
\mathrm{mg} / \mathrm{dL} \\
\text { Mujeres: } \leq 50 \mathrm{mg} / \mathrm{dL}\end{array}$ & $\begin{array}{l}\text { Hombres: } \leq 40 \\
\text { mg/dL } \dagger \\
\text { Mujeres: } \leq 50 \\
\text { mg/dL } †\end{array}$ & $\begin{array}{l}\text { Hombres: } \leq 40 \\
\text { mg/dL } \dagger \\
\text { Mujeres: } \leq 50 \mathrm{mg} / \mathrm{dL} \dagger\end{array}$ \\
\hline
\end{tabular}


Los datos fueron ingresados y analizados en el software SPSS v16. Se calculó la prevalencia global y por género de SMet en la población estudiada, con base en los criterios del ATP-III (1), la AHA (2) y la IDF (3). Adicionalmente, se calculó la prevalencia en hombres con base en la modificación del punto de corte de PA propuesto (IDF-m). Para corregir el efecto de selección por género, se realizó un pareamiento aleatorio hombre: mujer a un ratio de 1:1 (73 hombres y 73 mujeres). El análisis fue similar en los datos pareados y en el resto de los datos. Las diferencias de prevalencia por género y criterio utilizado fueron evaluadas mediante la prueba del Ji-cuadrado. Se consideraron diferencias estadísticamente significativas todas las pruebas pareadas con $\mathrm{p}<0,05$.

La concordancia entre cada uno de los criterios diagnósticos de SMet fue valorada mediante el test de kappa ( $\kappa)$ media de Cohen, con base en los parámetros propuestos por Landis y Koch, en los que si ê es menor o igual a 0,00 se considera que no hay concordancia, entre 0,01-0,20 la concordancia es pobre, entre 0,210,40 es ligera, entre $0,41-0,60$ es clara, entre $0,61-0,80$ es buena, entre $0,81-0,92$ es muy buena y si es mayor de 0,93 es considerada excelente (14).

\section{RESULTADOS}

De 324 pacientes examinados 77,5\% fueron mujeres. La edad promedio fue 63 años (DE $\pm 11,87)$ con rango entre 27 y 93 años y significativamente superior en varones (66 años vs 62,1 años) $p=0,01$. Las diferencias entre las mediciones promedio de tensión arterial sistólica y diastólica, perímetro abdominal, HDLc, triglicéridos y glucemia en ayunas no fueron estadísticamente significativas. En la Tabla 1 se detallan la edad y las diferencias por género de cada uno de los criterios para síndrome metabólico.

La prevalencia global fue de 59,9 \% (ATP III), 83,6 \% (AHA), 81,2 \% (IDF) y $92,9 \%$ (IDFm), con diferencias estadísticamente significativas en todas las comparaciones: ATP III-AHA, ATP III-IDF, ATP III-IDFm, AHA-IDF, AHAIDFm, IDF-IDFm ( $p<0,001$ en todas las anteriores comparaciones de prevalencia global).

Las diferencias entre varones y mujeres solo fueron estadísticamente significativas en las definiciones del ATP III, y el IDF (no modificado), ( $p<0,001$ para las dos definiciones) teniendo los varones una menor prevalencia. Una vez se actualizaron los parámetros diagnósticos del SMet, tanto del AHA y del IDFm, las diferencias se hicieron menos notorias y no estadísticamente significativas ( $\mathrm{p}=0,144, \mathrm{y} \mathrm{p}=0,346$, respectivamente). 
Tabla 1. Edad y criterios para síndrome metabólico en cada género

\begin{tabular}{|c|c|c|c|c|c|c|c|c|}
\hline \multirow{2}{*}{ Característica } & \multicolumn{2}{|c|}{$\begin{array}{l}\text { Masculino } \\
(\mathrm{n}: 73)\end{array}$} & \multicolumn{2}{|c|}{$\begin{array}{c}\text { Femenino } \\
(\mathrm{n}: 250)\end{array}$} & \multicolumn{2}{|c|}{$\begin{array}{c}\text { Total } \\
(n: 323)\end{array}$} & \multicolumn{2}{|c|}{ Diferencia de géneros } \\
\hline & Promedio & $\mathrm{SD}$ & Promedio & SD & Promedio & SD & $x^{2}$ & Valor de $p$ \\
\hline Edad & 66 & 12,8 & 62,1 & 11,4 & 63 & 11,9 & 6,28 & 0,013 \\
\hline TAS & 138 & 21 & 137 & 21 & 137 & 20,9 & 0,125 & 0,724 \\
\hline TAD & 81 & 10 & 79 & 10 & 76,6 & 9,7 & 2,195 & 0,139 \\
\hline PA & 95 & 14 & 97 & 12 & 96,9 & 12,6 & 1,30 & 0,254 \\
\hline HDLC & 46 & 9 & 49 & 33 & 48,4 & 29,5 & 0,807 & 0,37 \\
\hline TAG & 134 & 82 & 143 & 66 & 141 & 69,9 & 1,002 & 0,317 \\
\hline GEA & 92,5 & 34,2 & 102,3 & 39,9 & 100,1 & 38,8 & 3,68 & 0,056 \\
\hline
\end{tabular}

TAS: Tensión arterial sistólica en $\mathrm{mm} \mathrm{Hg}$; TAD: Tensión arterial diastólica en mm $\mathrm{Hg}$; PA: Perímetro abdominal en centímetros; TAG: Triglicéridos en ayunas en mg/dL; GEA: Glucosa en ayunas en mg/DI

Análisis de concordancia

La valoración de la concordancia reveló una pobre o ligera concordancia entre las definiciones del IDFm, comparadas con el ATP III y la AHA $(\kappa=0,147$, y $\kappa=0,299$, respectivamente). La mayor concordancia se encontró entre los criterios de la AHA y del IDF $(\kappa=0,638)$.

Las demás comparaciones (Tabla 2 ) fueron claras y entre los rangos 0,4-0,5. Los criterios del ATP III comparados con los del IDF mostraron un kappa de 0,405. Las comparaciones de criterios que se basaban de uno previo fueron claras: ATP III y AHA $(\kappa=0,496)$, e IDF y el IDFm $(\kappa=0,455)$.

Tabla 2. Concordancia en la definición de síndrome metabólico entre los criterios diagnósticos de la AHA, el ATP III, la IDF y su modificación propuesta para

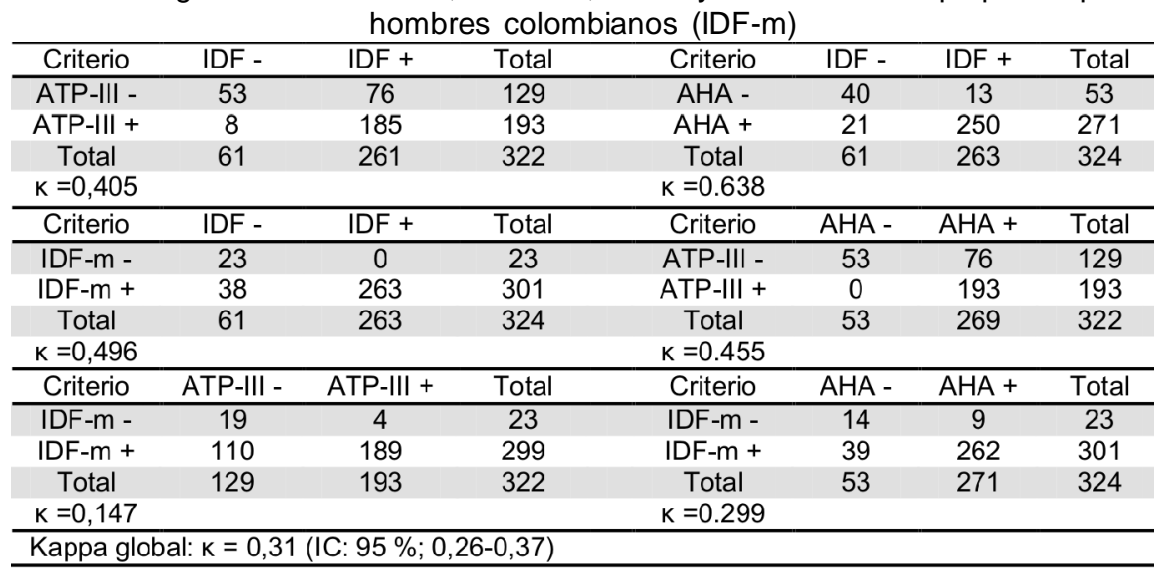


Emparejamiento hombres: mujeres

El emparejamiento hombres:mujeres no mostró diferencia en el test de kappa en comparación con los resultados de toda la base de datos (Tabla 3). Ninguna de las comparaciones de definiciones de SMet realizadas entre la base de datos total y el pareamiento por géneros demostraron diferencias estadísticamente significativas (ATP III: 0,491; AHA: 0,587; IDF: 0,200; IDFm: $0,710)$.

Tabla 3. Comparación de la prevalencia de síndrome metabólico entre cada una de las definiciones (emparejamiento hombres-mujeres a un ratio $1: 1$ )

\begin{tabular}{cccccc}
\hline \multicolumn{2}{c}{ Comparación. } & $\begin{array}{c}\text { Porcentajes } \\
(\%)\end{array}$ & $K$ & $\begin{array}{c}\text { Valor de } p \\
\text { (Diferencias entre } \\
\text { prevalencias) }\end{array}$ & $\begin{array}{c}\text { Valor de } p \\
\text { (Diferencias } \\
\text { entre kappas) }\end{array}$ \\
\hline ATP III & AHA & 56,6 vs. 85,6 & 0,361 & $<0,001$ & 0,233 \\
ATP III & IDF & 56,6 vs. 76,0 & 0,438 & $<0,001$ & 0,700 \\
ATP III & IDF-m & 56,6 vs. 93,8 & 0,127 & $<0,001$ & 0,747 \\
AHA & IDF & 85,6 vs. 76,0 & 0,608 & $<0,001$ & 0,762 \\
AHA & IDF-m & 85,6 vs. 93,8 & 0,270 & $<0,001$ & 0,830 \\
IDF & IDF-m & 76,0 vs. 93,8 & 0,345 & $<0,001$ & 0,169 \\
\hline * & Diferencias entre Kappas comparando las diferencias del pareamiento, y de toda la base de datos.
\end{tabular}

\section{DISCUSIÓN}

En Colombia, como en el resto de países en vía de desarrollo, se ha descrito un aumento de la morbilidad y mortalidad derivada de las enfermedades cardiovasculares durante los últimos treinta años, lo cual ha sido explicado por la creciente exposición a hábitos de vida altamente aterogénicos (sedentarismo, dieta hipergrasa, hipercalórica) (15-17); asícomo de los trastornos del metabolismo de los carbohidratos $(18,19)$. Estos factores explican por qué la prevalencia de SMet en este grupo de pacientes hipertensos fue superior a la descrita en grandes cohortes de países desarrollados de Asia $(20,21)$ y Europa $(12,21-24)$.

El estudio INTERHEART encontró que la prevalencia de hipertensión, diabetes mellitus fue superior en los países Latinoamericanos (incluyendo Colombia) en comparación con Estados Unidos (19). Aunque la obesidad central (definida según el índice cintura-cadera) fue mucho más prevalente en los países desarrollados de Norte América y Europa Occidental. Con la emergencia de la nueva definición internacional de la IDF (3) los estudios realizados en población general han demostrado que la prevalencia de SMet es superior a la referida con las definiciones de la ATP-III y su actualización en conjunto con la AHA $(7,25-$ 27). Sin embargo, en individuos que han padecidoeventos cardiovasculares (infarto agudo del miocardio, angina inestable, cirugía de revascularización coronaria o 
infarto cerebral) la definición que se utilice no afecta la capacidad de detección de ninguna de las definiciones (7).

Nuestros hallazgos sugieren que en la población hipertensa, tal como ocurre en quienes ya han padecido algún evento cardiovascular, la prevalencia global se modifica en función de la definición utilizada; lo cual también ha sido evidenciado en otro estudio realizado en Colombia en una población de pacientes hipertensos, donde describen una prevalencia de 27,3\% según los criterios del ATP III y de $75,9 \%$ según los de la $\mathrm{AHA}(13)$.

El análisis por géneros evidenció que en varones la prevalencia de SMet fue significativamente menor $(\mathrm{p}<0,05)$ con la utilización de los criterios del ATP III y del IDF. Esto sería explicable por una prevalencia menor de obesidad central en mujeres, ya que los demás parámetros son muy similares (Figura 1).

Figura 1. Porcentaje de SMet según las diferentes definiciones en hombres o mujeres

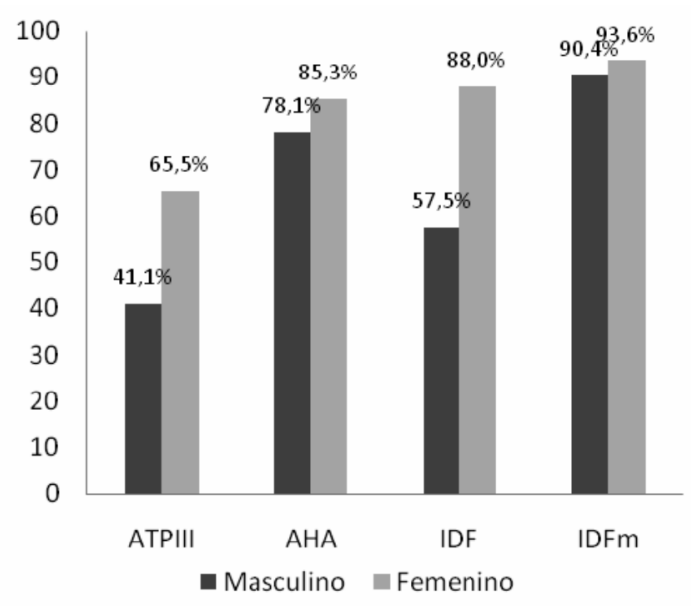

El análisis de la concordancia demostró una pobre correlación entre las comparaciones con el IDFm (excepto con el IDF). Esto demuestra que también en los pacientes hipertensos la modificación de la definición tiene un impacto en la prevalencia del SMet. Las demás correlaciones son claras, incluyendo el ATP III-IDF $(\kappa=0,405)$, ATP III-AHA $(\kappa=0,455)$, y la IDF-IDFm $(\kappa=0,496)$. La concordancia IDF-AHA fue la mejor $(\kappa=0,638)$, demostrando que la actualización de la definición del ATP III realizada en conjunción con la AHA tiene buena correlación con el criterio de la IDF en pacientes hipertensos, a pesar de sus diferencias. 
Algunos estudios retrospectivos $(11,28)$ sugieren que los criterios de la IDF clasifican muchas personas como enfermas, las cuales tienen un bajo riesgo de eventos cardiovasculares en comparación con los criterios del ATP-III. En un estudio realizado en Suecia se encontró que los pacientes hipertensos con SMet según la definición de la IDF no tienen mayor riesgo de eventos cardiovasculares OR (IC: $95 \%$; 0,86-1,44), contrario a aquellos identificados mediante las definiciones del ATP-III o de la AHA (28). Teniendo en cuenta que la definición de la IDF es más sensible (11), es probable que estos hallazgos se deban a su capacidad para detectar muchas personas en riesgo, antes de que desarrollen las complicaciones.

Consideramos que la detección de una mayor cantidad de pacientes hipertensos con SMet brinda la oportunidad para la implementación y optimización de estrategias para la prevención primaria y secundaria de la enfermedad cardiovascular, en especial cambios terapéuticos del estilo de vida $(9,29)$. Además de identificar los pacientes de alto riesgo, permite guiar el seguimiento, ya que es más probable que éstos requieran más fármacos anti-hipertensivos, tengan un menor control glicémico y de los lípidos séricos $(23,24)$; así como una mayor frecuencia de afectación renal, cardiaca y de los vasos cerebrales, tal como se demostró en el estudio ETODH (Evaluation of Target Organ Damage in Hypertension) (22).

La declaración de la Sociedad Europea de Hipertensión acerca del SMet en hipertensión arterial (30) recomienda la implementación sistemática de los cambios terapéuticos de los hábitos de vida (dieta, ejercicio físico, reducción de peso, etc.) y cuando sean necesarios fármacos anti-hipertensivos; preferir el uso de inhibidores de la enzima convertidora, bloqueadores de los receptores de angiotensina y/o calcio-antagonistas; ya que éstos disminuyen el riesgo de diabetes de nueva aparición; y evitar los diuréticos y beta-bloqueadores (especialmente su combinación) porque lo aumentan.

En síntesis, el análisis de concordancia demostró al menos, una clara concordancia la mayoría de las definiciones analizadas. Con una correlación pobre entre los criterios comparados con IDFm y un aumento estadísticamente significativo de la prevalencia de SMet con esta definición. La detección del SMet y su manejo, inicialmente con cambios terapéuticos de los hábitos de vida, es una oportunidad en la prevención primaria y secundaria de la enfermedad cardiovascular que debe ser aplicada sistemáticamente a todos los pacientes hipertensos, ya que presentan alto riesgo de complicaciones renales, cardiacas y cerebro-vasculares 
Agradecimientos: A Patricio López Jaramillo, M.D., Ph.D., por el apoyo en la consecución de la bibliografía.

Conflictos de interés: Ninguno.

\section{REFERENCIAS}

1. Executive Summary of The Third Report of The National Cholesterol Education Program (NCEP) Expert Panel on Detection, Evaluation, And Treatment of High Blood Cholesterol In Adults (Adult Treatment Panel III). JAMA 2001 May 16;285(19):2486-97.

2. Grundy SM, Cleeman JI, Daniels SR, Donato KA, Eckel RH, Franklin BA, et al. Diagnosis and management of the metabolic syndrome: an American Heart Association/National Heart, Lung and Blood Institute Scientific Statement. Circulation 2005 Oct 25;112(17):2735-52.

3. Alberti KG, Zimmet P, Shaw J. The metabolic syndrome-a new worldwide definition. Lancet 2005 Sep 24-30;366(9491):1059-62.

4. Alonso AL, Munguia-Miranda C, Ramos-Ponce D, Hernandez-Saavedra D, Kumate J, Cruz M. Waist perimeter cutoff points and prediction of metabolic syndrome risk. A study in a Mexican population. Arch Med Res 2008 Apr;39(3):346-51.

5. Barbosa PJ, Lessa I, de Almeida Filho N, Magalhaes LB, Araujo J. Criteria for central obesity in a Brazilian population: impact on metabolic syndrome. Arq Bras Cardiol 2006 Oct;87(4):407-14.

6. Garcia RG, Cifuentes AE, Caballero RS, Sanchez L, Lopez-Jaramillo P. A proposal for an appropriate central obesity diagnosis in Latin American population. Int $\mathrm{J}$ Cardiol 2006 Jun 16;110(2):263-4

7. Lopez-Jaramillo P, Rueda-Clausen CF, Silva FA. The utility of different definitions of metabolic syndrome in Andean population. Int J Cardiol 2007 Apr 4;116(3):421-2.

8. Perez M, Casas JP, Cubillos-Garzon LA, Serrano NC, Silva F, Morillo CA, et al. Using waist circumference as a screening tool to identify Colombian subjects at cardiovascular risk. Eur J Cardiovasc Prev Rehabil 2003 Oct;10(5):328-35.

9. Barrera MP, Pinilla AE, Cortés E, Mora G, Rodríguez MN. Síndrome metabólico: una mirada interdisciplinaria. Rev Col Cardiol 2008;15(3):111-26.

10. Hwu CM, Hsiung CA, Wu KD, Lee WJ, Shih KC, Grove J, et al. Diagnosis of insulin resistance in hypertensive patients by the metabolic syndrome: AHA vs. IDF definitions. Int J Clin Pract 2008 Sep;62(9):1441-6.

11. Limpawattana P, Sawanyawisuth K, Busaracome P, Foocharoen C, Phitsanuwong C, Chumjan S, et al. The best criteria to diagnose metabolic syndrome in hypertensive Thai patients. J Med Assoc Thai 2008 Apr;91(4):485-90.

12. Marboeuf P, Gras M, Rosey G, Fontaine P, Mounier-Vehier C. The metabolic syndrome: marker of cardiovascular risk in hypertensive patients? The importance of definition. Arch Mal Coeur Vaiss 2007 Aug;100(8):642-8.

13. Lombo B, Villalobos C, Tique C, Satizábal C, Franco C. Prevalencia del síndrome metabólico entre los pacientes que asisten al servicio de clínica de hipertensión de la Fundación Santa Fe de Bogotá. Rev Col Cardiol 2006;12(7):472-8.

14. Landis JR, Koch GG. The measurement of observer agreement for categorical data. Biometrics1977 Mar;33(1):159-74.

15. Lopez-Jaramillo P, Pradilla LP, Castillo VR, Lahera V. Socioeconomic pathology as a cause of regional differences in the prevalence of metabolic syndrome and pregnancyinduced hypertension. Rev Esp Cardiol 2007 Feb;60(2):168-78. 
16. Braguinsky J. Prevalencia de obesidad en América Latina. Anales Sis San Navarra 2002;25(Supl.1):109-15.

17. Rueda-Clausen CF, Silva FA, Lopez-Jaramillo P. Epidemic of overweight and obesity in Latin America and the Caribbean. Int J Cardiol 2008 Mar 28;125(1):111-2.

18. Aschner P. Diabetes trends in Latin America. Diabetes Metab Res Rev 2002 Sep-Oct;18 Suppl 3:S27-31.

19. Yusuf S, Hawken S, Ounpuu S, Dans T, Avezum A, Lanas F, et al. Effect of potentially modifiable risk factors associated with myocardial infarction in 52 countries (the INTERHEART study): case-control study. Lancet 2004 Sep 11-17;364(9438):93752.

20. Lee CM, Huxley RR, Woodward M, Zimmet P, Shaw J, Cho NH, et al. Comparisons of metabolic syndrome definitions in four populations of the Asia-Pacific region. Metab Syndr Relat Disord 2008 Spring;6(1):37-46.

21. Otero F, Grigorian L, Lado M, Lado A, Turrado V, Santos J, et al. Asociación entre hipertensión refractaria y riesgo cardiometabólico. Estudio HIPERFRE. Nefrología 2008;28(4):425-32.

22. Cuspidi C, Valerio C, Giudici V, Negri F, Sala C, Zanchetti A, et al. Metabolic syndrome and multiple organ damage in essential hypertension. Blood Press 2008;17(4):195-203.

23. Kjeldsen SE, Naditch-Brule L, Perlini S, Zidek W, Farsang C. Increased prevalence of metabolic syndrome in uncontrolled hypertension across Europe: the Global Cardiometabolic Risk Profile in Patients with hypertension disease survey. J Hypertens 2008 Oct;26(10):2064-70.

24. Rodilla E, Garcia L, Merino C, Costa JA, Gonzalez C, Pascual JM. Impact of metabolic syndrome in the control of blood pressure and dyslipemia. Med Clin (Barc) 2004 Nov 6;123(16):601-5.

25. Athyros VG, Ganotakis ES, Elisaf MS, Liberopoulos EN, Goudevenos IA, Karagiannis A. Prevalence of vascular disease in metabolic syndrome using three proposed definitions. Int J Cardiol 2007 Apr 25;117(2):204-10.

26. Manzur F, Alvear C, Alayón A. Caracterización fenotípica y metabólica del síndrome metabólico en Cartagena de Indias. Rev Col Cardiol 2008;15(3):97-101.

27. Pinzon JB, Serrano NC, Diaz LA, Mantilla G, Velasco HM, Martinez LX, et al. Impact of the new definitions in the prevalence of the metabolic syndrome in an adult population at Bucaramanga, Colombia. Biomedica 2007 Jun;27(2):172-9.

28. Nilsson PM, Engstrom G, Hedblad B. The metabolic syndrome and incidence of cardiovascular disease in non-diabetic subjects-a population-based study comparing three different definitions. Diabet Med 2007 May;24(5):464-72.

29. Mendivil CO, Cortes E, Sierra ID, Ramírez A, Molano LM, Tovar LE, et al. Reduction of global cardiovascular risk with nutritional versus nutritional plus physical activity intervention in Colombian adults. Eur J Cardiovasc Prev Rehabil 2006 Dec;13(6):947-55.

30. Redon J, Cifkova R, Laurent S, Nilsson P, Narkiewicz K, Erdine S, et al. The metabolic syndrome in hypertension: European society of hypertension position statement. J Hypertens 2008 Oct;26(10):1891-900. 